\title{
Quasiadiabatic dynamics of ultracold bosonic atoms in a one-dimensional optical superlattice
}

\author{
A. Dhar, ${ }^{1}$ D. Rossini, ${ }^{2}$ and B. P. Das ${ }^{3}$ \\ ${ }^{1}$ COMP Centre of Excellence and Department of Applied Physics, Aalto University, FI-00076 Aalto, Finland \\ ${ }^{2}$ NEST, Scuola Normale Superiore and Istituto Nanoscienze-CNR, I-56126 Pisa, Italy \\ ${ }^{3}$ Indian Institute of Astrophysics, II Block, Koramangala, Bangalore 560 034, India
}

(Received 24 August 2014; revised manuscript received 9 June 2015; published 11 September 2015)

\begin{abstract}
We study the quasiadiabatic dynamics of a one-dimensional system of ultracold bosonic atoms loaded in an optical superlattice. Focusing on a slow linear variation in time of the superlattice potential, the system is driven from a conventional Mott insulator phase to a superlattice-induced Mott insulator, crossing in between a gapless critical superfluid region. Due to the presence of a gapless region, a number of defects depending on the velocity of the quench appear. Our findings suggest a power-law dependence similar to the Kibble-Zurek mechanism for intermediate values of the quench rate. For the temporal ranges of the quench dynamics that we considered, the scaling of defects depends nontrivially on the width of the superfluid region.
\end{abstract}

DOI: 10.1103/PhysRevA.92.033610

PACS number(s): 67.85.-d, 03.75.Nt, 05.10.Cc, 05.30.Jp

\section{INTRODUCTION}

Ultracold atomic gases in optical lattices provide a unique platform to probe a wide range of quantum phenomena with a high degree of controllability. The success of the BoseHubbard (BH) Hamiltonian [1,2] in elucidating the behavior of ultracold bosons in optical lattices has stimulated a great deal of interest from both theoretical and experimental points of view. In particular, the seminal work on the Mott insulator (MI) to superfluid (SF) quantum phase transition (QPT) [3] paved the way for a number of studies that led to the observation of various exotic quantum phases $[4,5]$.

Nonequilibrium quantum effects in such systems can be investigated by varying in time parameters such as the optical lattice depth or the magnetic field close to a Feshbach resonance. Such experimental possibilities have spurred renewed interest in the study of both sudden and quasiadiabatic quenches [6,7]. The latter would provide important insights into nonequilibrium quantum phase transitions. In the presence of a ground-state energy gap that is always finite during a very slow time evolution, the adiabatic theorem guarantees that the system will remain in the instantaneous ground state of the corresponding time-dependent Hamiltonian. However, if a gapless region is crossed, the system will be unable to stay in its equilibrium ground state, regardless of how slowly it is quenched. The nonadiabatic evolution inevitably excites the system, and a number of defects will appear in the evolved state. The mechanism of such defect formation was first addressed by Kibble and Zurek (KZ) in the context of classical phase transitions in the early universe $[8,9]$ and was more recently extended to the quantum regime for the case of adiabatic quenches across a single quantum critical point $[10,11]$.

The possibility to apply this kind of quench has led to a number of theoretical studies addressing different types of many-body systems, including spin chains and ultracold quantum gases (see, e.g., Refs. [12-33]). Despite the large body of work in this field, several aspects involving the response of such systems to slow quenches have not been completely understood and deserve further investigation. It is believed that, in the presence of nonisolated critical points or of extended critical regions, the validity of the $\mathrm{KZ}$ mechanism is a priori not obvious, even if in some cases it is still possible to predict the defect density by identifying a dominant critical point or by using scaling arguments $[20,22,23]$.

The dynamics of ultracold bosons in an optical lattice subjected to a quasiadiabatic quench has been theoretically analyzed for the MI-SF transition as well as the reverse transition [12,14]. The SF-MI transition for a slow quench has also been studied while taking the effects of the parabolic trapping potential into account [30]. These results highlighted the emergence of a scaling behavior for the characteristic length scale as a function of the quench rate, which is well approximated by a power law. However, it has been shown that, for a phase transition of the Kosterlitz-Thouless type (as is the case for the MI-SF transition in the one-dimensional BH model), the exponents depend on that rate and are generally different from the KZ prediction based on the critical exponents that are relevant for asymptotically long quench times [33]. Experimental evidence supporting the growth of the condensate excitations with a power-law dependence on the quench rate has been observed for the MI-SF transition for ultracold bosons in an optical lattice [31]. Furthermore, for the same transition in a similar system, the observation of the emergence of coherence and a power-law dependence of the correlation length on the quench rate for intermediate quenches has recently been reported [34].

The feasibility of superposing different optical lattices with distinct frequencies [35] also made the study of local relaxation dynamics possible in such superlattice setups [36,37]. An interesting property of these composite structures when they are loaded with bosonic atoms is that they can facilitate the generation of multiple lattice-modulated MI phases, which can isolate SF regions in the parameter space of the system $[38,39]$. To the best of our knowledge, slow quenches for QPTs in optical superlattices starting and ending in insulating phases and crossing a superfluid region in between have not been addressed so far, and this is the focus of our present work.

Here we consider a one-dimensional (1D) system of ultracold bosonic atoms loaded in an optical superlattice, formed by two superimposed optical standing waves with different frequencies. At zero temperature, this system exhibits different quantum phases: MI, SF, and a superlattice-induced MI (SLMI) with periodically modulated on-site occupa- 
tion [38,39]. The superlattice potential is constructed to vary linearly in time and is chosen in such a way that it crosses a gapless region in between two insulating regions. We consider the formation of defects in the final state after the quench and demonstrate a nontrivial scaling of the excess energy as a function of the quench rate. We tackle this problem by means of the time-dependent density-matrix renormalization group (DMRG) method in the formalism of a matrix-product-state ansatz [40].

This paper is organized as follows. We start by introducing our model and discussing the static properties of its groundstate phase diagram that are relevant to the ongoing discussion (Sec. II). In Sec. III we define our dynamical protocol and discuss the formation of defects and the behavior of two-point correlation functions at the end of the protocol. Finally, in Sec. IV we draw our conclusions.

\section{MODEL AND PHASE DIAGRAM}

The model is described by the following Hamiltonian:

$$
\hat{\mathcal{H}}_{\mathrm{SLBH}}=\sum_{i}-J\left(\hat{a}_{i}^{\dagger} \hat{a}_{i+1}+\text { H.c. }\right)+\frac{U}{2} \hat{n}_{i}\left(\hat{n}_{i}-1\right)+\lambda_{i} \hat{n}_{i},
$$

where $\hat{a}_{i}^{\dagger}, \hat{a}_{i}$ denote the creation and annihilation operators on site $i$ satisfying the usual bosonic commutation rules, with $\hat{n}_{i}=\hat{a}_{i}^{\dagger} \hat{a}_{i}$ being the corresponding number operator. The parameter $J$ denotes the hopping amplitude, $U$ is the onsite repulsive interaction strength, and $\lambda_{i}$ quantifies the superlattice potential depth. For the period-two optical superlattice that we have considered, $\lambda_{i}$ has a finite value of $\lambda>0$ for odd sites, and it is zero for even sites. Hereafter we work in units of $\hbar=1$ and set $J=1$ as the energy scale. The critical $U$ value for the MI-SF transition is located at $U_{c} \approx 3.3$ for $\lambda=0$ [41], as shown in Fig. 1.

The ground-state phase diagram of model (1) has been studied by means of mean-field theory [38], quantum Monte Carlo techniques [42], and the DMRG method [39]. In one dimension and at integer filling $\bar{n}=1$, this is given in the $\lambda-U$ plane as in Fig. 1. Here we identify the various phases by analyzing the behavior of the ground-state energy gap $\Delta$ as a function of the system size in the following way. First, we observe that, for a MI, the energy gap is finite and coincides with the charge gap $\Delta_{+}-\Delta-$, which is the difference between the energy cost to add $\left(\Delta_{+}\right)$and to remove $\left(\Delta_{-}\right)$a particle from the system. On a chain of finite length $L$, the numerical evaluation of the Mott gap has been thus obtained by performing three DMRG iterations, with projections on different number sectors $L, L \pm 1$. The corresponding ground states respectively give the desired energies $E_{0}, E_{ \pm}=E_{0}+\Delta_{ \pm}$. In the $\mathrm{SF}$ region, this gap vanishes as the inverse of the system size $L$. The critical points have been extracted as those in which the product $\Delta \times L$ for the smallest $(L=140)$ and the largest $(L=200)$ considered lengths differed more than $4 \%$ (see the insets of Fig. 1).

The nature of the insulating phase (MI or SLMI) depends on the relative strength of $U$ and $\lambda$. A SF region is present in between these two insulators, and its width decreases with increasing $U$. The intervening SF phase arises because of the competition between the superlattice potential $\lambda$ and the on-site

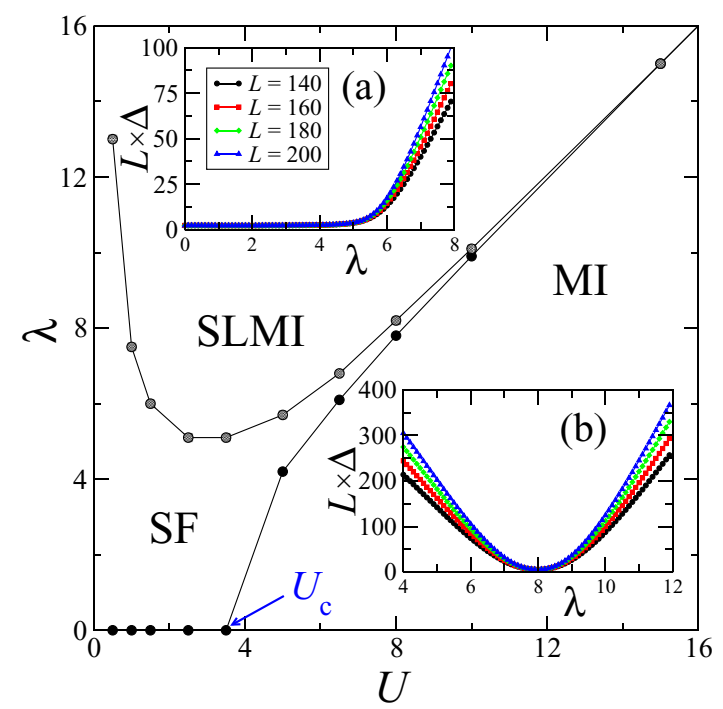

FIG. 1. (Color online) Zero-temperature phase diagram in the $\lambda-U$ plane of the Hamiltonian $\hat{\mathcal{H}}_{\mathrm{SLBH}}$ for a period-two optical superlattice and with unitary filling. Data have been obtained from DMRG calculations of the charge gap, which is the energy for adding or removing a particle from the state with $\bar{n}=1$. The insets show the ground-state energy gaps as a function of $\lambda$ and for different system sizes and two values of $U$, (a) smaller than $U_{c}(U=2.5)$ and (b) larger than $U_{c}(U=8.0)$. The gaps in the SF region close as the inverse system size $L^{-1}$. Here and in the subsequent figures the Hamiltonian parameters $\lambda$ and $U$ as well as the energy gaps $\Delta$ are expressed in units of $J$.

two-body interaction $U$. For $U<U_{c}$, there is only a transition from a gapless SF to a gapped SLMI at a critical value of $\lambda$. But for $U>U_{c}$, there are three possible scenarios. If $\lambda$ is much smaller than $U$, the system remains in the MI phase. When it becomes comparable to $U$, the system makes a transition to the SF phase, and for large values of $\lambda$ it enters the SLMI phase.

\section{QUASIADIABATIC QUENCH DYNAMICS}

In order to probe the slow quench dynamics of ultracold atoms in an optical superlattice, Eq. (1), it is necessary to analyze the excitations that are generated when the gapless SF phase is crossed. In view of the specific features of the phase diagram, it is convenient to fix a value of $U$ and increase the parameter $\lambda$ to drive the system across the MI-to-SF and then the SF-to-SLMI phase transitions. As can be seen in Fig. 1, the width of the critical region changes with $U$. This reflects a nontrivial dependence of the rate of defect generation with $U$, as discussed later.

We adopt a linear variation in time of the superlattice potential $\lambda$, which is given by

$$
\lambda(t)=\lambda_{0}+\left(\lambda_{f}-\lambda_{0}\right) t / \tau .
$$

Here $\tau$ denotes the time of the quench, while $\lambda_{0}$ and $\lambda_{f}=$ $\lambda(\tau)$ are, respectively, the initial and the final values of the superlattice potential. After fixing the value of $U$, we choose $\lambda_{0}$ and $\lambda_{f}$ such that the system starts from a MI phase and ends in a SLMI phase (except for the cases with $U<U_{c}$, where there 

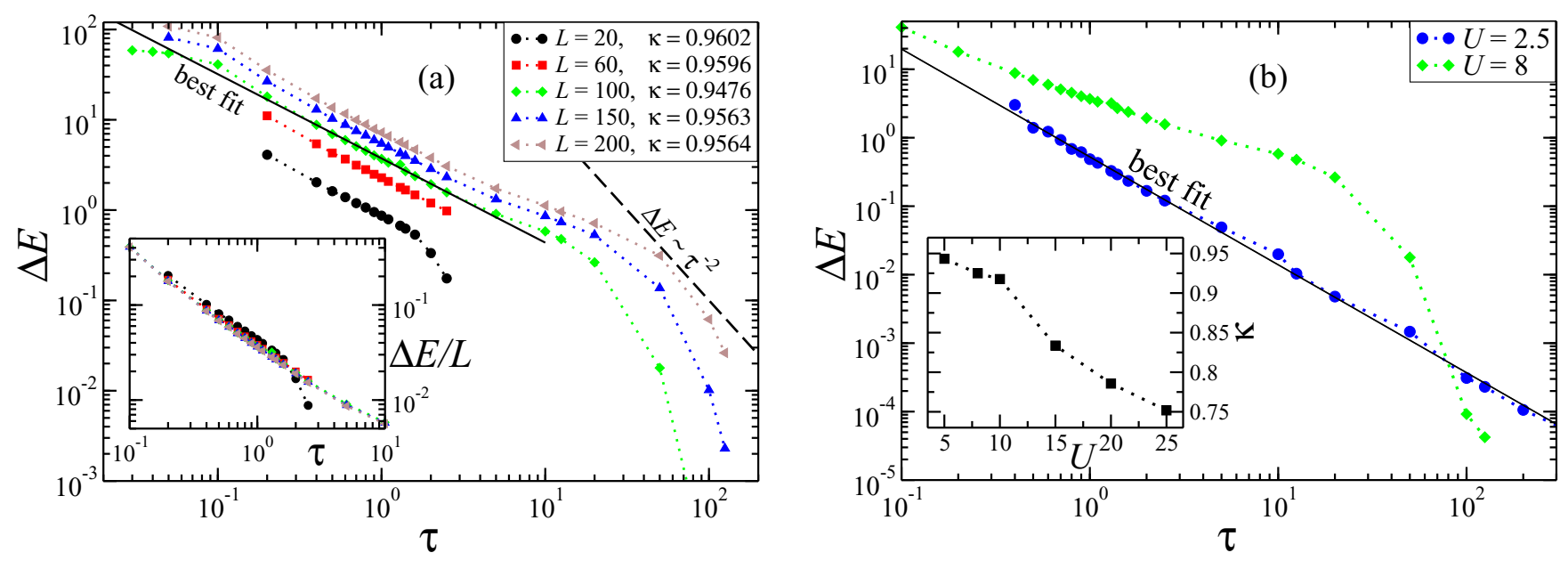

FIG. 2. (Color online) Excess energy $\Delta E(\tau)$ at the end of the time evolution as a function of the quench time $\tau$. (a) Quench from the MI to SLMI phase, with a SF phase in between. Here we fix $U=8$ and $\lambda: 5.5 \rightarrow 10.5$. The various data sets stand for different system lengths. Our fitting procedure in the intermediate region gives the values of $\kappa$ as shown in the legend. The inset shows same data rescaled over the size $L$. (b) Quench from the SF to SLMI phase (blue circles: $U=2.5, \lambda: 0 \rightarrow 5, L=100$ ); the green diamonds show the same set as that reported in (a). The solid straight line is a power-law fit of the DMRG data and gives a value of $\kappa \approx 1.5754$. The inset shows the power-law decay rate $\kappa$ for the excess energy in the intermediate scaling region as a function of the on-site interaction $U$ for quenches across the MI $\rightarrow$ SF $\rightarrow$ SLMI phase transitions $\left(U>U_{c}\right)$. Here and in the subsequent figures times are expressed in units of $\hbar / J$, while excess energies $\Delta E$ are given in units of $J$.

is no MI phase). In between the initial and the final insulating phases, there is a SF region, whose width depends on $U$. Due to the presence of a gapless region at the thermodynamic limit $L \rightarrow \infty$, a certain number of defects in the final state after the evolution will appear, no matter how slowly the quenching is performed [43]. Below we shed light on these defects.

The system wave function $|\psi(t)\rangle$ evolves according to the time-dependent Schrödinger equation. We computed $|\psi(\tau)\rangle$ at the final time $\tau$ after the quench (2), using a time-evolving block-decimation strategy [40,44]. We simulated systems up to $L=200$ sites with no more than $n_{\max }=3$ bosons per site and used open boundary conditions. The time interval $[0, \tau]$ has been discretized into many slices of time length $\Delta t \ll 1$, where $\hat{\mathcal{H}}(t)$ is assumed to be constant. The corresponding time evolution operator $e^{-i \hat{\mathcal{H}}(t) \Delta t}$ has been expanded by means of a sixth-order Suzuki-Trotter decomposition [45]. We have been able to consider a time step $\Delta t=0.05$ and reach a threshold for the discarded states $\varepsilon=10^{-9}$ by using a bond-link dimension $m \lesssim 200$ for all our simulations [46].

\section{A. Excess energy}

To quantify the defect generation due to the nonadiabatic crossing of the SF region during the time evolution, we focus on the residual energy $\Delta E(t)$, defined as the excess energy above the ground state:

$$
\Delta E(t)=\langle\psi(t)|\hat{\mathcal{H}}(t)| \psi(t)\rangle-\left\langle\psi_{\mathrm{GS}}(t)|\hat{\mathcal{H}}(t)| \psi_{\mathrm{GS}}(t)\right\rangle,
$$

where $\langle\psi(t)|\hat{\mathcal{H}}(t)| \psi(t)\rangle$ denotes the energy of the system at time $t$, while $\left\langle\psi_{\mathrm{GS}}(t)|\hat{\mathcal{H}}(t)| \psi_{\mathrm{GS}}(t)\right\rangle$ is the instantaneous ground-state energy for Hamiltonian $\hat{\mathcal{H}}_{\mathrm{SLBH}}$ at time $t$. This quantity serves as an analog of the defect density originally considered by Kibble and Zurek (see, e.g., Refs. [18-20]). Let us now discuss its behavior after a time modulation of the superlattice depth $\lambda(t)$ from $t=0$ to $t=\tau$, as dictated by Eq. (2). In particular we focus on the scaling of $\Delta E(\tau)$ with $\tau$ for different values of interaction $U$.

The typical scenario is depicted in Fig. 2(a), where we are able to distinguish three distinct behaviors as a function of the quench time. For $\tau \gg 1$, the dynamics is ruled by the adiabatic regime typical of slow quenches: the time-evolved wave function remains very close to the instantaneous ground state of $\hat{\mathcal{H}}(t)$. The residual energy follows a power-law behavior

$$
\Delta E \sim \tau^{-\kappa}
$$

with $\kappa=2$. This exponent can be obtained within the LandauZener formalism [47], where the quantum evolution is studied by means of an effective two-level approximation with an avoided level crossing. We point out that the adiabatic regime can occur only in the presence of an instantaneous ground-state energy gap which remains finite along the sweeping (2) (i.e., for quenches much slower than the inverse square of the minimum crossed gap, $\tau \gg \Delta_{\min }^{2}$ ). Therefore this is a behavior related to a finite-size effect, which disappears at the thermodynamic limit where the gap in the superfluid region is rigorously zero. In the opposite regime of fast quenches ( $\tau \ll 1)$, the dynamics is strongly nonadiabatic, and the initial state is essentially frozen during the evolution. The excess energy thus saturates with $\tau$. The intermediate regime in between is the most interesting one since it is crucially affected by the critical properties of the region crossed by the system.

In the intermediate regime, our data display a power-law scaling of the type in Eq. (4). This fairly agrees with the general behavior predicted by KZ and verified in many cases when the system is adiabatically driven across isolated quantum critical points [6]. The KZ mechanism roughly identifies two types of evolution, either adiabatic or impulsive, according to the distance from the critical point. The time (distance from the 
critical point) at which the system switches to the impulsive regime depends on the quench velocity. This simple argument indeed predicts a power-law scaling form for $\Delta E$ as a function of $\tau$, with a rate $\kappa$ expressed in terms of the critical exponents dictating the phase transition. However, for the crossing of continuous phase transitions with extended critical regions, as is in our case, the $\mathrm{KZ}$ scaling may still give insightful information but cannot be regarded as ultimately predictive. In the specific case of a Kosterlitz-Thouless transition analogous to that occurring in the $\mathrm{BH}$ model at $\lambda=0$, it has been shown that the exponentially slow gap closure induces a power-law scaling which generally depends on the quench rate. The exponent differs from that obtained with the usual $\mathrm{KZ}$ mechanism using the critical exponents of the transition [33]. In our specific situation, the system is quenched from a gapped to a gapless phase and then to another gapped phase, thus crossing two QPTs and an extended critical (SF) region (Fig. 1, for fixed $U>U_{c}$ ). This is an even more complex scenario, in which it is impossible to grasp the quantitative power-law scaling predicted by $\mathrm{KZ}$, and hence we expect the emergence of a more complex and inhomogeneous behavior in terms of the size of the crossed critical region.

In the case of $U=8$ we clearly identify an intermediate scaling region with $\kappa \approx 0.95(1)$, as extracted from fits of the numerical data [see the solid line in Fig. 2(a), denoting the best fit of the data series at the size $L=100$ ]. Our results do not display a significant dependence of $\kappa$ on $L$. Notice also that the excess energy per unit length is universal in the scaling region, whose width increases with $L$, due to the gap closure in the SF phase [inset of Fig. 2(a)]. A qualitatively similar behavior is observed for a quench from the SF to SLMI phase at fixed $U<U_{c}$; Fig. 2(b) (blue circles) shows an example with $U=$ 2.5. The value $\kappa \approx 1.58$ in the intermediate region obtained for that case is considerably larger than that for $U=8$.

These observations support the evidence that any appropriate scaling analysis should depend nontrivially on $U$, while a simple $\mathrm{KZ}$ argument cannot predict this complex behavior. We point out that it is also not guaranteed that $\kappa$ does not change with the quench rate, as theoretically predicted for the MI-SF transition at $\lambda=0$ [33]. In the range of $\tau$ we were able to address, we did not observe such a dependence. However, as shown in the inset of Fig. 2(b), for quenches across the phases $\mathrm{MI} \rightarrow \mathrm{SF} \rightarrow$ SLMI with $U>U_{c}$, we found a rather complex dependence of $\kappa$ on $U$ and hence on the width of the intermediate SF region. In particular the defect production rate $\kappa$ decreases monotonically as a function of the time during which the system is crossing the gapless region.

We also checked the dependence of $\kappa$ on the starting and ending points inside the insulator (we varied $\lambda_{i}$ and $\lambda_{f}$ for fixed $U$ ). Results in Fig. 3 indicate a tendency toward a slight decrease of $\kappa$ if the gapped region crossed by the quench increases.

\section{B. Correlation functions}

Finally, we examined the behavior of the two-point correlation function $C(r)=\left\langle\psi(\tau)\left|\hat{a}_{i}^{\dagger} \hat{a}_{j}\right| \psi(\tau)\right\rangle$ at the end of the quasiadiabatic dynamics and observed an exponential decay with the distance $r=|i-j|$, as shown in Fig. 4. The two points $i$ and $j$ have been chosen in a symmetric way with

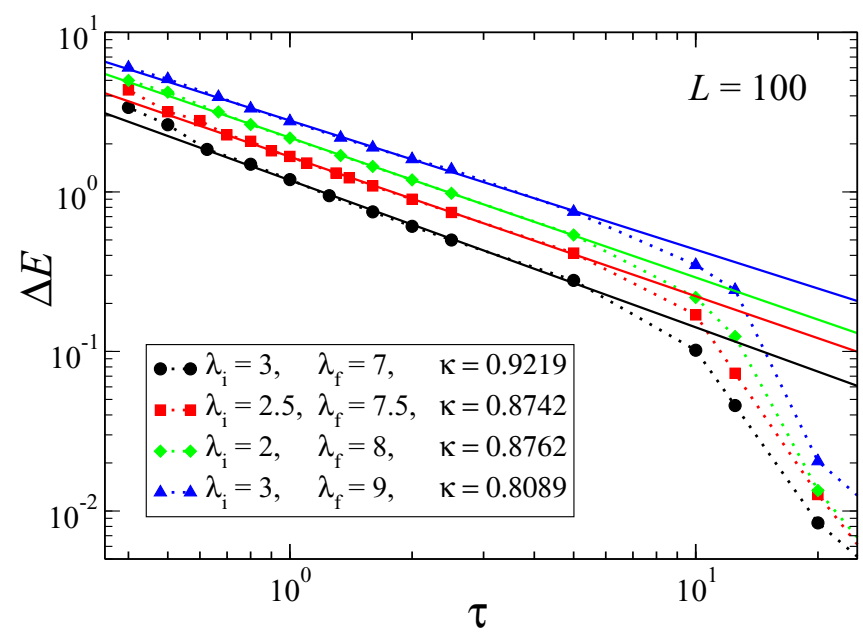

FIG. 3. (Color online) Excess energy as a function of the quenching time for fixed $U=5$ and a system size $L=100$ for different initial and final points.

respect to the center of the chain in order to minimize boundary effects, such that $i=(L-r+1) / 2, j=(L+r+1) / 2$ for odd $r$ and $i=(L-r) / 2, j=(L+r) / 2$ for even $r$ (for instance, for $L=100$ sites, $r=1$ corresponds to $i=50, j=$ $51 ; r=2$ corresponds to $i=49, j=51 ; r=3$ corresponds to $i=49, j=52$; and so on).

In the inset we plot the correlation function

$$
\xi=\sqrt{\frac{\sum_{r} r^{2}\left\langle\hat{a}_{i}^{\dagger} \hat{a}_{j}\right\rangle}{\sum_{r}\left\langle\hat{a}_{i}^{\dagger} \hat{a}_{j}\right\rangle}}, \quad r=|i-j| .
$$

This clearly exhibits a nonmonotonic behavior as a function of the quench rate $\tau$ [48]. In particular we notice that $\xi(\tau)$ is increasing initially in the intermediate scaling region. This

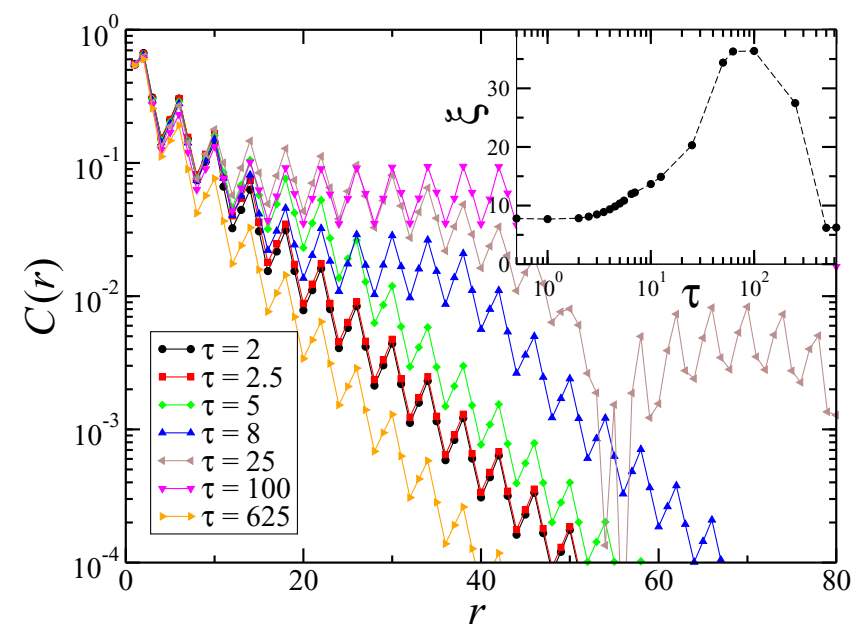

FIG. 4. (Color online) Two-point correlation function $C(r)=$ $\left\langle\psi(\tau)\left|\hat{a}_{i}^{\dagger} \hat{a}_{j}\right| \psi(\tau)\right\rangle$ as a function of the distance $r=|i-j|$ and for different quench times $\tau$. The inset shows the correlation length $\xi$ as extracted from numerical data as a function of $\tau$. Data are for $U=8$ and $\lambda: 5.5 \rightarrow 10.5$. Here we set $L=100$. Distances are in units of the lattice spacing. 
can be attributed to the persistence of quasi-long-range order which the system gained while quenching through the SF phase. But after some critical value of $\tau$, we observe that $\xi$ starts to decrease. Such a behavior is ascribed to the onset of the adiabatic regime. Since the system ends up in an insulating phase (SLMI), where the correlation function decays exponentially, we expect the correlation length to be small in the adiabatic regime. In particular, the value of $\tau$ at which the transition from the intermediate regime to the adiabatic regime takes place, obtained from the analysis of the residual energy (Fig. 2), coincides with that seen for the correlation length (Fig. 4).

\section{SUMMARY}

We have theoretically analyzed the slow quench dynamics of ultracold bosonic atoms in a one-dimensional optical superlattice. By considering a linear time dependence of the superlattice potential, we showed that, when crossing a gapless superfluid region, the system has the tendency to generate defects. This fact is due to the adiabaticity loss during the time evolution, which occurs even when the system is quenched very slowly. Our results show a complex dependence of the rate of defect generation on the quench velocity, which cannot be understood in terms of the Kibble-Zurek physics underlying the crossing of a single critical point.
From an experimental point of view, the behavior of the excess energy could be verified by means of time-of-flight measurements of the correlation length. This, in turn, may reveal itself to be a simple indicator of the presence or absence of the power-law scaling regime for the defect production as a function of the quench velocity. Trapping ultracold bosonic atoms in optical standing waves is probably the most favorable setup to probe this kind of physics. Recent experiments have already verified the power-law behavior [31,34] in the framework of the Bose-Hubbard model. Moreover, the capability that has been demonstrated by a variety of outof-equilibrium experiments with great accuracy and for large coherence times, ranging from sudden quenches to adiabatic variation of the system's parameters, puts our results arising from the quenching of the superlattice potential in a favorable position for verification in the laboratory.

\section{ACKNOWLEDGMENTS}

We thank R. Fazio for useful discussions. A.D. and B.P.D. thank D. Sen and A. Dutta for stimulating discussions. A.D. acknowledges the Academy of Finland through its Center of Excellence Programme (2012-2017) and under Project No. 13251748. D.R. acknowledges support from the Italian MIUR through FIRB Project No. RBFR12NLNA. The computations were performed on the Intel Cluster at the Indian Institute of Astrophysics.
[1] M. P. A. Fisher, P. B. Weichman, G. Grinstein, and D. S. Fisher, Phys. Rev. B 40, 546 (1989).

[2] D. Jaksch, C. Bruder, J. I. Cirac, C. W. Gardiner, and P. Zoller, Phys. Rev. Lett. 81, 3108 (1998).

[3] M. Greiner, O. Mandel, T. Esslinger, T. W. Hänsch, and I. Bloch, Nature (London) 415, 39 (2002); M. Greiner, O. Mandel, T. W. Hänsch, and I. Bloch, ibid. 419, 51 (2002).

[4] M. Lewenstein, A. Sanpera, V. Ahufinger, B. Damski, A. Sen(De), and U. Sen, Adv. Phys. 56, 243 (2007).

[5] I. Bloch, J. Dalibard, and W. Zwerger, Rev. Mod. Phys. 80, 885 (2008).

[6] J. Dziarmaga, Adv. Phys. 59, 1063 (2010).

[7] A. Polkovnikov, K. Sengupta, A. Silva, and M. Vengalattore, Rev. Mod. Phys. 83, 863 (2011).

[8] T. W. B. Kibble, J. Phys. A 9, 1387 (1976).

[9] W. H. Zurek, Nature (London) 317, 505 (1985).

[10] W. H. Zurek, U. Dorner, and P. Zoller, Phys. Rev. Lett. 95, 105701 (2005).

[11] A. Polkovnikov, Phys. Rev. B 72, 161201(R) (2005).

[12] S. R. Clark and D. Jaksch, Phys. Rev. A 70, 043612 (2004).

[13] J. Dziarmaga, Phys. Rev. Lett. 95, 245701 (2005).

[14] R. Schützhold, M. Uhlmann, Y. Xu, and U. R. Fischer, Phys. Rev. Lett. 97, 200601 (2006).

[15] R. W. Cherng and L. S. Levitov, Phys. Rev. A 73, 043614 (2006).

[16] F. M. Cucchietti, B. Damski, J. Dziarmaga, and W. H. Zurek, Phys. Rev. A 75, 023603 (2007).

[17] L. Cincio, J. Dziarmaga, M. M. Rams, and W. H. Zurek, Phys. Rev. A 75, 052321 (2007).
[18] T. Caneva, R. Fazio, and G. E. Santoro, Phys. Rev. B 76, 144427 (2007).

[19] T. Caneva, R. Fazio, and G. E. Santoro, Phys. Rev. B 78, 104426 (2008).

[20] F. Pellegrini, S. Montangero, G. E. Santoro, and R. Fazio, Phys. Rev. B 77, 140404(R) (2008).

[21] K. Sengupta, D. Sen, and S. Mondal, Phys. Rev. Lett. 100, 077204 (2008).

[22] S. Deng, G. Ortiz, and L. Viola, Europhys. Lett. 84, 67008 (2008).

[23] U. Divakaran, A. Dutta, and D. Sen, Phys. Rev. B 78, 144301 (2008).

[24] A. Polkovnikov and V. Gritsev, Nat. Phys. 4, 477 (2008).

[25] C. De Grandi, R. A. Barankov, and A. Polkovnikov, Phys. Rev. Lett. 101, 230402 (2008).

[26] E. Canovi, D. Rossini, R. Fazio, and G. E. Santoro, J. Stat. Mech. (2009) P03038.

[27] V. Mukherjee and A. Dutta, Europhys. Lett. 92, 37004 (2010).

[28] M. Haque and F. E. Zimmer, Phys. Rev. A 87, 033613 (2013).

[29] C. Trefzger and K. Sengupta, Phys. Rev. Lett. 106, 095702 (2011).

[30] J.-S. Bernier, G. Roux, and C. Kollath, Phys. Rev. Lett. 106, 200601 (2011); J.-S. Bernier, D. Poletti, P. Barmettler, G. Roux, and C. Kollath, Phys. Rev. A 85, 033641 (2012).

[31] D. Chen, M. White, C. Borries, and B. DeMarco, Phys. Rev. Lett. 106, 235304 (2011).

[32] D. Pertot, A. Sheikhan, E. Cocchi, L. A. Miller, J. E. Bohn, M. Koschorreck, M. Köhl, and C. Kollath, Phys. Rev. Lett. 113, 170403 (2014). 
[33] J. Dziarmaga and W. H. Zurek, Sci. Rep. 4, 5950 (2014).

[34] S. Braun, M. Friesdorf, S. S. Hodgman, M. Schreiber, J. P. Ronzheimer, A. Riera, M. del Rey, I. Bloch, J. Eisert, and U. Schneider, Proc. Natl. Acad. Sci. USA 112, 3641 (2015).

[35] S. Fölling, S. Trotzky, P. Cheinet, M. Feld, R. Saers, A. Widera, T. Müller, and I. Bloch, Nature (London) 448, 1029 (2007).

[36] M. Cramer, A. Flesch, I. P. McCulloch, U. Schollwöck, and J. Eisert, Phys. Rev. Lett. 101, 063001 (2008); A. Flesch, M. Cramer, I. P. McCulloch, U. Schollwöck, and J. Eisert, Phys. Rev. A 78, 033608 (2008).

[37] S. Trotzky, Y.-A. Chen, A. Flesch, I. P. McCulloch, U. Schollwöck, J. Eisert, and I. Bloch, Nat. Phys. 8, 325 (2012).

[38] A. Dhar, M. Singh, R. V. Pai, and B. P. Das, Phys. Rev. A 84, 033631 (2011).

[39] A. Dhar, T. Mishra, R. V. Pai, and B. P. Das, Phys. Rev. A 83, 053621 (2011).

[40] U. Schollwöck, Ann. Phys. (NY) 326, 96 (2011).

[41] T. D. Kühner, S. R. White, and H. Monien, Phys. Rev. B 61, 12474 (2000).

[42] V. G. Rousseau, D. P. Arovas, M. Rigol, F. Hebert, G. G. Batrouni, and R. T. Scalettar, Phys. Rev. B 73, 174516 (2006).
[43] Note that, by first taking the thermodynamic limit $L \rightarrow \infty$, we are guaranteed that the system in the SF phase will be gapless; therefore the evolution will never be adiabatic, and defects will necessarily be formed at any finite value of $\tau$. On the contrary, by first taking the asymptotically slow quench limit $\tau \rightarrow \infty$, the evolution will stay adiabatic at any size $L$, and defects will not form.

[44] G. Vidal, Phys. Rev. Lett. 93, 040502 (2004).

[45] M. Suzuki, Commun. Math. Phys. 51, 183 (1976); H. F. Trotter, Proc. Am. Math. Soc. 10, 545 (1959).

[46] We have checked that, on the scale of all the figures presented here, our results do not present significant deviations if we take a time step $\Delta t=0.025$ corresponding to half of the value used throughout the simulations shown in this paper. This produces a discrepancy in the decay rates $\kappa$ which turns out to be less than $0.5 \%$.

[47] C. Zener, Proc. R. Soc. London, Ser. A 137, 696 (1932); L. D. Landau and E. M. Lifshitz, Quantum Mechanics (Pergamon, London, 1958), Vol. 3.

[48] The two summations in Eq. (5) have been performed neglecting ten lattice sites per border to reduce their influence (i.e., we considered $r=1, \ldots, L-20)$. 